\title{
Stress Cardiac Magnetic Resonance Imaging
}

National Cancer Institute

\section{Source}

National Cancer Institute. Stress Cardiac Magnetic Resonance Imaging. NCI Thesaurus. Code C80409.

A study using magnetic resonance to observe cardiac activity during the administration of pharmacologic stressors. 